\title{
Rejection rates for multiple-part manuscripts
}

\author{
David M. Schultz
}

Received: 6 March 2010/Published online: 11 June 2010

(C) Akadémiai Kiadó, Budapest, Hungary 2010

\begin{abstract}
Multiple-part manuscripts are those submitted to a journal and intended for publication as a series, usually having "Part 1," "Part I," ... "Part $N$ " in the title. Although some journals prohibit such submissions, other journals (including Monthly Weather Review) have no such restrictions. To examine how reviewers and editors view multiplepart manuscripts, 308 multiple-part manuscripts submitted to Monthly Weather Review from May 2001 through February 2010 were examined. For multiple-part manuscripts having reached a final decision, 67\% were accepted, which was also the average acceptance rate of all manuscripts $(67 \%)$. Part I manuscripts submitted alone had a lower acceptance rate $(61 \%)$ than the average, whereas Part II manuscripts submitted alone had a higher acceptance rate $(77 \%)$ than the average. Two-part manuscripts submitted together had an acceptance rate (67\%) comparable to the average. Typical reviewer comments for Part I manuscripts submitted alone included the manuscript being too long for the available results and the author making claims in Part I that would be supported in the unseen Part II. Typical comments for Part II manuscripts submitted alone included the somewhat contradictory statements that material was unnecessarily duplicated in the two manuscripts and more repetition was needed between the two parts. For two-part manuscripts submitted together, reviewers often recommended condensing the two manuscripts and merging them into one. In some cases, editors rejected manuscripts even though no reviewer recommended rejection because the sum of all reviewers' comments would require substantial reorganization of the manuscripts. The results of this study suggest the following recommendations for authors considering writing multiple-part manuscripts: Write manuscripts that are sensibly independent of each other, make minimal reference to unsubmitted
\end{abstract}

\section{M. Schultz}

Division of Atmospheric Sciences, Department of Physics, University of Helsinki, Helsinki, Finland

D. M. Schultz (西)

Finnish Meteorological Institute, P.O. Box 503, Erik Palménin Aukio 1, 00101 Helsinki, Finland e-mail: David.Schultz@fmi.fi

D. M. Schultz

Centre for Atmospheric Science, School of Earth, Atmospheric and Environmental Sciences, University of Manchester, Manchester, UK 
manuscripts, and have sufficient and substantiated scientific content within each manuscript.

Keywords Multiple-part manuscripts $\cdot$ Rejection rate $\cdot$ Reviewers ·

Editors

\section{Introduction}

Manuscripts with "Part 1," "Part I,"..., "Part $N$ " in the title that are intended to be published as a series are called multiple-part manuscripts. Some journals require that all parts of a multiple-part manuscript be submitted together (e.g., Adsorption, Concepts in Magnetic Resonance Part A, Journal of Environmental Informatics); others require statements to the editor on why the work requires two parts (e.g., International Journal of Impact Engineering); others discourage multiple-part manuscripts (e.g., Journal of Neuroscience); and yet others prohibit them (e.g., International Journal of Computer Mathematics).

I serve as the Chief Editor for Monthly Weather Review (MWR), which is one of ten scientific journals published by the American Meteorological Society. None of these ten journals have a policy on the submission of multiple-part manuscripts. In 2008, the American Meteorological Society published 134 multiple-part articles (identified by searching for "part" in the title), 36 of which were in MWR (12\% of the total 299 articles published). The popularity of multiple-part papers has not diminished-in 1998, 86 articles were published in American Meteorological Society journals with "part" in the title, 28 of which were in $M W R$ (15\% of the total 189 articles published). The number of multiple-part papers in American Meteorological Society journals seems to have increased dramatically starting in the mid 1960s, although earlier multiple-part papers do exist among the Society's publications (e.g., Starr Malkus and Stern 1953; Stern and Starr Malkus 1953).

Despite the popularity of multiple-part articles in American Meteorological Society journals (as well as other journals), no one seems to have taken a look at this form of publication. Specifically, what are the advantages and disadvantages of multiple-part manuscripts from the varied perspectives of authors, editors, reviewers, and readers?

Authors may write multiple-part manuscripts for several reasons. First, multiple-part manuscripts allow a common theme to be identified among a series of papers, usually originating from a single research group. As an example, one of the biggest proponents of multiple-part papers was the late Prof. Peter Hobbs of the University of Washington with his 16-part series in Journal of the Atmospheric Sciences "The Mesoscale and Microscale Structure and Organization of Clouds and Precipitation in Midlatitude Cyclones" (19801988), his 7-part series in Journal of the Atmospheric Sciences and MWR "Organization and Structure of Clouds and Precipitation on the Mid-Atlantic Coast of the United States" (1989-2002), and his 6-part series in $M W R$ "Structure and Evolution of Winter Cyclones in the Central United States and their Effects on the Distribution of Precipitation" (19952000). A second reason why authors may choose multiple-part manuscripts is that authors may find that they have too much material for a single manuscript, and so turn their research into a multiple-part manuscript.

Despite these advantages to authors, multiple-part manuscripts can be troublesome for editors (Sect. 3.3 in Schultz 2009a). Finding reviewers is difficult enough; reviewers are even less enthusiastic about reviewing multiple-part manuscripts (most multiple-part manuscripts submitted together to $M W R$ are handled by the same reviewers, although there 
is no rule that they need to be), and many reviewers will take longer to deliver the reviews, slowing down the peer-review process. Another problem happens when multiple-part manuscripts arrive sequentially rather than together. What happens if one of the manuscripts gets accepted, but the other is rejected? Part II may have to wait until Part I is accepted.

Although some readers may be pleased that multiple-part manuscripts organize an author's oeuvre on a particular topic, other readers may be annoyed at not knowing whether reading Part I is necessary to understand Part II. Some readers may fear that to read one means to read them all in order to understand what was done. Moreover, some Part I papers are still waiting for their Part II papers to get published (e.g., Ellingson and Gille 1978; Semtner and Holland 1980), some Part II papers advertised a submitted Part III that was never published (e.g., Droegemeier and Wilhelmson 1985), or there may be a long time between publication of the parts (e.g., Davies-Jones 1991, 2009). Unpublished subsequent manuscripts may be frustrating to readers, who want to see what came next or may be searching endlessly for the unpublished companion paper.

To understand what scientists think of multiple-part papers, Schultz (2010b) conducted a survey of attendees at the 14th Cyclone Workshop and students in a scientific communication skills course at the University of Helsinki. One of the questions on the survey was "I believe multipart manuscripts (Part I, Part II, etc.) are acceptable." More experienced authors at the Cyclone Workshop (professors, scientists, and forecasters) believed that multiple-part manuscripts were acceptable in most cases (54\%) compared to $41 \%$ of early career attendees (students and postdoctoral fellows) and only $25 \%$ of the University of Helsinki students (Fig. 1). The early career attendees at the Cyclone Workshop and students at the University of Helsinki were also more likely to say that multiple-part manuscripts were rarely acceptable (6 and $16 \%$, respectively; Fig. 1).

Although this survey provides some indication of the perceptions of authors and readers, this survey was able to assess perceptions only hypothetically. A more concrete demonstration of how scientists assess multiple-part manuscripts can be derived from studying the peer-review process of a scientific journal. Therefore, this article studies the fate of 308 multiple-part manuscripts submitted to $M W R$ over nearly 9 years. Reviewer recommendations (e.g., accept, revisions required, reject) and final acceptance and rejection rates are examined for these manuscripts in "Multiple-part manuscripts submitted to Monthly Weather Review" section. In "What the reviewers said" section, a subset of 94 manuscripts are examined where the individual reviews are available for analysis. "Recommendations" section provides recommendations for authors considering multiple-part manuscripts, and "Conclusion" section summarizes this article.

\section{Multiple-part manuscripts submitted to Monthly Weather Review}

To examine how multiple-part papers fare in the review process, all multiple-part manuscripts that have been recorded in the American Meteorological Society's Manuscript Tracking System were collected by searching titles for the word "part." This approach may miss the occasional title where the "part" is omitted, such as "Remote Sounding of High Clouds. V: Infrared Properties and Structures of Tropical Thunderstorm Anvils," but that seems to be a small number of manuscripts. This search produced 308 manuscripts received at $M W R$ between 4 May 2001 and 28 February 2010. Of these 308 manuscripts, $198(64 \%)$ were accepted, 83 (27\%) were rejected, 13 (4\%) were withdrawn, $11(4 \%)$ had not received a final decision, and $2(0.7 \%)$ were transferred to another journal. Thus, of the 


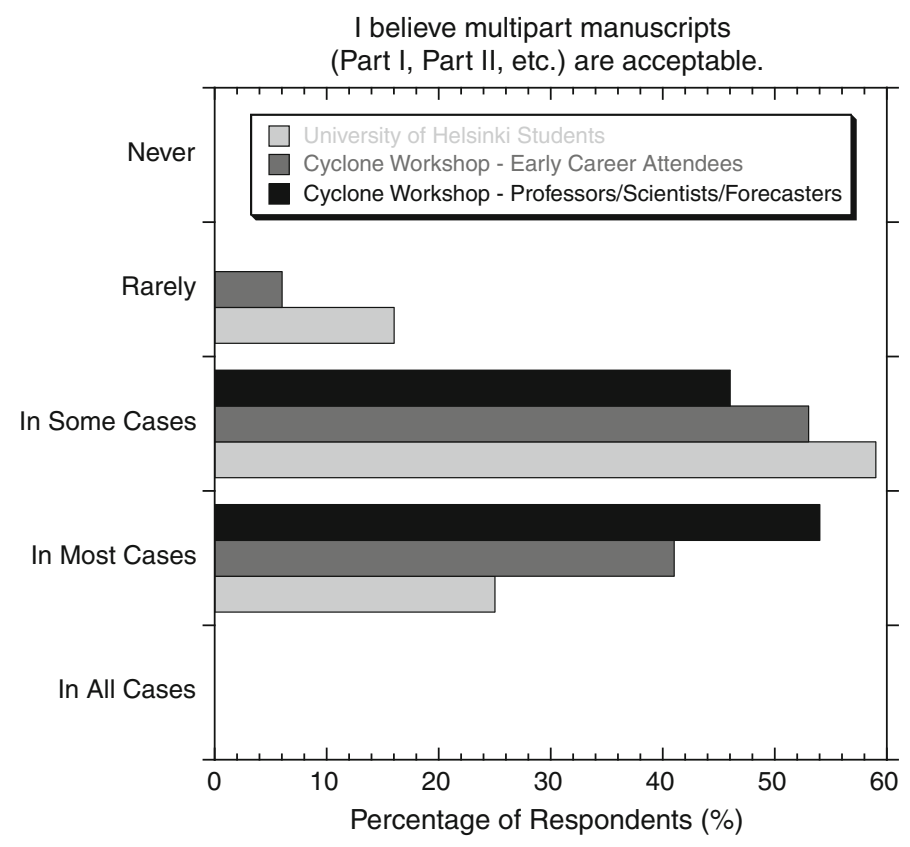

Fig. 1 Percentage of respondents to the question, "I believe multipart manuscripts (Part I, Part II, etc.) are acceptable" from three groups: 32 University of Helsinki students enrolled in a Communications Skills for Scientists course, 17 early-career attendees (students and postdoctoral fellows) of the 14th Cyclone Workshop, and 26 professor, scientist, and forecaster attendees of the 14th Cyclone Workshop. Data from Schultz (2010b)

297 multiple-part manuscripts for which $M W R$ made final decisions, 67\% were accepted and $33 \%$ were rejected, transferred, or withdrawn, which matches the results of a separate study of all 500 manuscripts during a 15.5-month period in 2007-2008 (Schultz 2009b; Table 1c). (During this almost 9-year period, the average annual acceptance rate for $M W R$ decreased from $72 \%$ in 2001 to $63 \%$ in 2009.) Thus, at first glance, the acceptance rate of multiple-part manuscripts is not different from the average for all manuscripts during this period.

These 297 manuscripts were separated into those that were submitted together and those that were submitted separately. Five principal groups were identified (Table 1): 61 Part I manuscripts that were submitted alone, 39 Part II manuscripts that were submitted alone, 168 two-part manuscripts (84 pairs) that were submitted together (within a month of each other), 7 Part III manuscripts that were submitted alone, and 15 three-part manuscripts (five trios) that were submitted together. One Part IV manuscript, one Part V manuscript, and one Part VI manuscript were each submitted alone, and one-four-part manuscript (quartet) was submitted together.

For the 61 Part I manuscripts that were submitted alone, $61 \%$ were accepted, $36 \%$ were rejected, and 3\% were withdrawn (Table 1a). In comparison, the 39 Part II manuscripts that were submitted independently of their companions had an acceptance rate of $77 \%$, rejection rate of $13 \%$, and withdrawal rate of $10 \%$ (Table 1a). Furthermore, of the 168 twopart manuscripts that were submitted together, $67 \%$ were accepted, $27 \%$ were rejected, and $4 \%$ were withdrawn (Table 1a). Given an average acceptance rate of $67 \%$ for $M W R$ (Schultz 2009b, 2010a; Table 1c), these results indicate a lower-than-average acceptance 
Table 1 Statistics for (a) two-part manuscripts and (b) three-part manuscripts submitted to MWR from May 2001 to February 2010 ("Multiple-part manuscripts submitted to Monthly Weather Review" section) compared to (c) the statistics for 500 manuscripts from 13 January 2007 to 2 May 2008 (Schultz 2009b)

\begin{tabular}{|c|c|c|c|c|c|c|}
\hline Category & $\begin{array}{l}\text { Number of } \\
\text { manuscripts }\end{array}$ & $\begin{array}{l}\% \\
\text { accepted }\end{array}$ & $\begin{array}{l}\% \\
\text { rejected }\end{array}$ & $\begin{array}{l}\% \\
\text { withdrawn }\end{array}$ & $\begin{array}{l}\text { Number } \\
\text { of } \\
\text { reviews }\end{array}$ & $\begin{array}{l}\% \text { reviews } \\
\text { recommending } \\
\text { rejection }\end{array}$ \\
\hline
\end{tabular}

(a) Two-part manuscripts

$\begin{array}{lrrrrrr}\text { Part I submitted alone } & 61 & 61 & 36 & 3 & 139 & 20 \\ \text { Part II submitted alone } & 39 & 77 & 13 & 10 & 80 & 9 \\ \begin{array}{l}\text { Two-part manuscripts } \\ \text { submitted together }\end{array} & 168 & 67 & 27 & 4 & 407 & 16 \\ \begin{array}{l}\text { (b) Three-part manuscripts } \\ \text { Part III submitted alone }\end{array} & 7 & 86 & 14 & 0 & & \\ \begin{array}{l}\text { Three-part manuscripts } \\ \text { submitted together }\end{array} & 15 & 33 & 67 & 0 & 29 & 41 \\ \begin{array}{l}\text { (c) } M W R \text { average } \\ \text { Average for } M W R \\ \text { manuscripts (Schultz }\end{array} & & & & & & \\ \text { 2009b) } & 500 & 67 & 30 & 3 & 1150 & 18\end{array}$

rate for Part I manuscripts submitted alone, but a higher-than-average acceptance rate for Part II manuscripts submitted alone. The average acceptance rate for two-part manuscripts submitted together is not different from the average for all manuscripts (Table 1a, c).

Of the 7 Part III manuscripts submitted alone, 6 (86\%) were accepted, whereas of the 15 Part III manuscripts submitted together, 5 (33\%) were accepted (Table 1b). Although the sample size was small, these results suggest that if the authors could get the first two parts submitted and accepted, then the third part would have a much greater chance for success in peer review. In contrast, submitting three parts together often ended up poorly, with $67 \%$ being rejected or withdrawn. Interestingly, of the seven manuscripts identified as Parts IV, $\mathrm{V}$, or VI, all were accepted for publication, including the quartet of manuscripts (Parts IIV) that were submitted together.

Of the 84 pairs of manuscripts submitted together, 54 pairs $(64 \%)$ had both manuscripts accepted; 25 pairs $(30 \%)$ had both manuscripts rejected, withdrawn or transferred; and 5 pairs $(6 \%)$ had one manuscript accepted and the other rejected or withdrawn. Of the fivethree-part manuscripts submitted together, one trio (20\%) had all three manuscripts accepted, three trios $(60 \%)$ had all three manuscripts rejected, and one trio (20\%) had two manuscripts accepted and one withdrawn.

\section{What the reviewers said}

The second phase of this research was to examine the initial reviews of multiple-part manuscripts. Not all reviews for all manuscripts were archived in the online Manuscript Tracking System, however. Thus, only 242 reviews of 94 manuscripts are examined in this section.

For Part I manuscripts submitted alone, 57 reviews of 23 manuscripts were available (Table 2). Of these, 18 reviews of 13 manuscripts specifically mentioned something about the manuscript being part of a multiple-part series, with 8 of those reviews on 7 
Table 2 What the reviewers said and the outcome of the manuscripts: 242 reviews of 94 manuscripts ("What the reviewers said" section)

\begin{tabular}{llllll}
\hline Category & $\begin{array}{l}\text { Number of } \\
\text { reviews on } \\
\text { number of } \\
\text { manuscripts (in } \\
\text { parentheses) }\end{array}$ & $\begin{array}{l}\text { Number of reviews on } \\
\text { number of manuscripts } \\
\text { (in parentheses) } \\
\text { mentioning multiple-part } \\
\text { manuscript }\end{array}$ & $\begin{array}{l}\text { Number and } \\
\text { percentage of } \\
\text { reviews } \\
\text { recommending } \\
\text { rejection }\end{array}$ & $\begin{array}{l}\text { Rejection } \\
\text { rate of } \\
\text { manuscripts } \\
(\%)\end{array}$ & $\begin{array}{l}\text { Withdrawal } \\
\text { rate of } \\
\text { manuscripts } \\
(\%)\end{array}$ \\
\hline $\begin{array}{l}\text { Part I } \\
\text { submitted } \\
\text { alone }\end{array}$ & $57(23)$ & $18(13)$ & $15(26 \%)$ & 43 & 4 \\
$\begin{array}{l}\text { Part II } \\
\text { submitted } \\
\text { alone }\end{array}$ & $38(17)$ & $18(12)$ & $6(15 \%)$ & 18 & 12 \\
$\begin{array}{c}\text { Two-part } \\
\text { manuscripts } \\
\text { submitted } \\
\text { together }\end{array}$ & $147(27$ pairs $)$ & $48(24$ pairs $)$ & $15(10 \%)$ & 30 & 4 \\
\hline
\end{tabular}

manuscripts recommending the papers be combined or submitted together, despite the reviewers not having seen subsequent parts. Relative to all manuscripts, more reviews recommended rejection and more manuscripts were rejected. Specifically, fifteen of the 57 reviews (26\%) recommended rejection, and $10(43 \%)$ of the manuscripts were eventually rejected and one (4\%) was withdrawn (Table 2). A sampling of some of the comments that were received follows. For example, some reviewers specifically mentioned that the manuscript was too long for the available results or disliked the author making claims in Part I that would be supported in an unseen Part II, a tactic that one reviewer said was "unfair to the reader." Another reviewer said, "Without Part II in front of me, I cannot consider the work as a whole." Yet another reviewer complained, "It is not even clear that [Part II] is a real paper. If it is real, it appears most of the real science will be in Part II."

For Part II manuscripts submitted alone, 38 reviews of 17 manuscripts were available (Table 2). Of these, 18 reviews of 12 manuscripts specifically mentioned the manuscript being part of a multiple-part series. Although more reviewers commented on the multiple-part nature of these Part II manuscripts compared to the Part I manuscripts submitted alone, fewer reviewers recommended rejection and fewer manuscripts were rejected. Specifically, six reviews (15\%) of four manuscripts recommended rejection of Part II, and three (18\%) manuscripts were rejected and two (12\%) were withdrawn (Table 2). Three reviews of three manuscripts wanted access to Part I (which they did not necessarily have). For example, some reviewers specifically mentioned flaws in Part I that were carried over into Part II, or that there was no value in Part II compared to Part I. Other reviews were somewhat contradictory with four reviews of four manuscripts wanting the two papers to stand more independently ("I really dislike things like, '...same conditions as were used in Part I." "), and six reviews of five manuscripts mentioning the need to reduce redundant material that was included in both parts. The contradictory reviews indicate one of the most serious concerns with multiple-part manuscripts: the difficulty in pleasing all reviewers - and by extension, all readers-by how the content within the two manuscripts is split.

For two-part manuscripts submitted together, 147 reviews of 27 manuscript pairs were available (Table 2). Of these, 48 reviews of 24 pairs specifically mentioned something 
about the manuscript being part of a multiple-part series, with 15 of those reviews on 12 pairs recommending that the papers be combined, a point one reviewer mentioned "might maximize the chance that people will read all of the authors' ideas." Fifteen reviews (10\%) recommended rejection of either part, and $16(30 \%)$ manuscripts were rejected and two (4\%) were withdrawn (Table 2). The much higher rejection rate for manuscripts compared to that for the reviewers' recommendations indicates that, although individual reviewers may not have recognized fatal flaws in the manuscripts, when all reviews of both manuscripts were assessed together, the editor decided that the large effort to reorganize the manuscripts would result in a substantially different manuscript. (Rejection is generally the preferred way for editors to handle such manuscripts at $M W R$.) For example, some reviewers specifically mentioned that flaws in Part I were carried over into Part II ("The misguided application of that equation already discussed in my review of Part I, is, as expected, repeated here."), that organization problems existed about what material should be in each part ("The results of data assimilation are used in Part I, but the data-assimilation technique is not described until Part II"), and that the scientific contribution of each part was not clear ("The introduction for Part II is also too short, without science background as well. Since the main foci of Part I and Part II are different, the introduction for each part should correspond to its own focus. Separate but comprehensive introductions should be given to both Part I and Part II."). Interestingly, many comments said that Part I was the stronger paper, and suggested merging the best material from Part II into a revised Part I ("this Part II is essentially an addendum to Part I", or "There is no theoretical advance here, merely the application of an established technique in a new situation. I suggest that the papers be significantly abbreviated and combined."). Given that some multiple-part papers originate as authors realize that they have too much material for a single manuscript and begin writing a second, such comments are perhaps not surprising. Alternatively, two manuscripts on related topics simply may wear out the reviewers.

\section{Recommendations}

Unlike some journals and publishers, $M W R$ and its publisher the American Meteorological Society have no formal policy on multiple-part manuscripts. Multiple-part manuscripts can be submitted together or independently of each other. The results of this research indicate that rejection rates are not substantially higher for multiple-part manuscripts, suggesting that the penalty is not substantially more severe for submitting multiple-part manuscripts. Nevertheless, authors may still face an uphill battle in convincing reviewers of the necessity for multiple-part manuscripts. Reviewers find multiple-part manuscripts challenging to review, and they often convey negative opinions, recommending eliminating text, combining multiple-part manuscripts, and recommending a different balance of material among the multiple-part manuscripts. One of the biggest challenges for authors is deciding whether to include information in one or both manuscripts. In some cases, a single reviewer will comment that some material should appear in both the manuscripts whereas other duplicated material should be included in only one manuscript. Such comments seem rather arbitrary, and an author might be hard pressed to second-guess what material should be duplicated and what should not be. Thus, for many authors, multiple-part manuscripts are more difficult to write because the balance between individual manuscripts and the series as a whole is more challenging than a single stand-alone manuscript. 
The results of this article suggest a strategy for authors considering writing multiple-part manuscripts.

1. Write non-multiple-part manuscripts that are independent of each other, and submit them independently. Doing so eliminates many reviewer complaints about multiplepart manuscripts. Furthermore, editors will have an easier time finding reviewers for the manuscript and decisions will be made more quickly. Also, issues with the data, methods, results, and interpretations can be clarified, resolved, or corrected in the review process, before subsequent manuscripts relying on such data, methods, results, and interpretations are submitted.

2. Make minimal references to upcoming papers. Despite an author's best intentions to finish a manuscript, sometimes plans change and a planned subsequent manuscript never materializes in print.

3. If the research is perceived to be too long for a single manuscript, then do not split without any clear rationale for how to organize the resulting manuscripts. Writing a single long, but complete, manuscript is better than thoughtlessly splitting one into two shorter, but incomplete or redundant, manuscripts.

4. If, after having considered all of the above, an author still wants to submit multiplepart manuscripts, the outcome at $M W R$ is somewhat mixed. Submitting manuscripts independently results in the first part having a lower chance of success, but the subsequent parts having a higher chance of success. Submitting them together produces results no different than the average for all manuscripts. Submitting them together also avoids negative reviews requesting information in the still-to-besubmitted second part that is unavailable to the reviewers.

5. Authors need to ensure that any multiple-part manuscripts avoid problems common to such submissions. Specifically, these problems include too much dependence between manuscripts and inadequate scientific content within each manuscript, problems that are both addressed by submitting independent manuscripts, as recommended in \#1-\#3 above.

By following these five recommendations, authors can hope to improve their chances for success in the peer-review process.

\section{Conclusion}

To see if the acceptance rate of multiple-part manuscripts differed from that of all manuscripts, this article examined 308 multiple-part manuscripts submitted to Monthly Weather Review from May 2001 to February 2010. Although Part I manuscripts submitted alone had a lower acceptance rate (61\%) than the average (67\%), Part II manuscripts submitted alone had a higher acceptance rate (77\%) than the average. On the other hand, two-part manuscripts submitted together had an acceptance rate (67\%) comparable to the average. For authors considering writing multiple-part manuscripts, the results of this study and the types of comments provided by reviewers suggest the following recommendations. Write independent manuscripts, do not reference unsubmitted research, and provide adequate evidence for claims within the manuscripts.

Acknowledgment Partial funding for Schultz comes from Vaisala Oyj. 


\section{References}

Davies-Jones, R. (1991). The frontogenetical forcing of secondary circulations. Part I: The duality and generalization of the $\mathbf{Q}$ vector. Journal of Atmospheric Sciences, 48, 497-509.

Davies-Jones, R. (2009). The frontogenetical forcing of secondary circulations. Part II: Properties of $\mathbf{Q}$ vectors in exact linear solutions. Journal of Atmospheric Sciences, 66, 244-260.

Droegemeier, K. K., \& Wilhelmson, R. B. (1985). Three-dimensional numerical modeling of convection produced by interacting thunderstorm outflows. Part II: Variations in vertical wind shear. Journal of Atmospheric Sciences, 42, 2404-2414.

Ellingson, R. G., \& Gille, J. C. (1978). An infrared radiative transfer model. Part 1: Model description and comparison of observations with calculations. Journal of Atmospheric Sciences, 35, 523-545.

Schultz, D. M. (2009a). Eloquent science: A practical guide to becoming a better writer, speaker, and atmospheric scientist (412 pp.). American Meteorological Society.

Schultz, D. M. (2009b). Are three heads better than two? How the number of reviewers and editor behavior affect the rejection rate. Scientometrics. doi:10.1007/s11192-009-0084-0.

Schultz, D. M. (2010a). Rejection rates for journals publishing atmospheric science. Bulletin of the American Meteorological Society, 91, 231-243.

Schultz, D. M. (2010b). A university laboratory course to improve scientific communication skills. Bulletin of the American Meteorological Society, 91. doi:10.1175/2010BAMS3037.1.

Semtner, A. J., \& Holland, W. R. (1980). Numerical simulation of equatorial ocean circulation. Part I: A basic case in turbulent equilibrium. Journal of Physical Oceanography, 10, 667-693.

Starr Malkus, J., \& Stern, M. E. (1953). The flow of a stable atmosphere over a heated island, part I. Journal of Meteorology, 10, 30-41.

Stern, M. E., \& Starr Malkus, J. (1953). The flow of a stable atmosphere over a heated island, part II. Journal of Meteorology, 10, 105-120. 